\title{
ITGA3 and ITGB4 expression biomarkers estimate the risks of locoregional and hematogenous dissemination of oral squamous cell carcinoma
}

\author{
Masaki Nagata ${ }^{1 *}$, Arhab A Noman ${ }^{1}$, Kenji Suzuki ${ }^{2}$, Hiroshi Kurita ${ }^{3}$, Makoto Ohnishi ${ }^{4}$, Tokio Ohyama $^{4}$, \\ Nobutaka Kitamura ${ }^{5}$, Takanori Kobayashi ${ }^{1}$, Kohya Uematsu' ${ }^{1}$, Katsu Takahashi ${ }^{6}$, Naoki Kodama ${ }^{1}$, Tomoyuki Kawase ${ }^{7}$, \\ Hideyuki Hoshina', Nobuyuki Ikeda', Susumu Shingaki ${ }^{8}$ and Ritsuo Takagi ${ }^{1}$
}

\begin{abstract}
Background: Molecular biomarkers are essential for monitoring treatment effects, predicting prognosis, and improving survival rate in oral squamous cell carcinoma. This study sought to verify the effectiveness of two integrin gene expression ratios as biomarkers.

Methods: Gene expression analyses of integrin a3 (ITGA3), integrin $\beta 4$ (ITGB4), CD9 antigen (CD9), and plakoglobin (JUP) by quantitative real-time PCR were conducted on total RNA from 270 OSCC cases. The logrank test, Cox proportional hazards model, and Kaplan-Meier estimates were performed on the gene expression ratios of ITGA3/ CD9 and ITGB4/JUP and on the clinicopathological parameters for major clinical events.

Results: A high rate (around 80\%) of lymph node metastasis was found in cases with a high ITGA3/CD9 ratio (high-ITGA3/CD9) and invasive histopathology (YK4). Primary site recurrence (PSR) was associated with high-ITGA3/CD9, T3-4 (TNM class), and positive margin, indicating that PSR is synergistically influenced by treatment failure and biological malignancy. A high ITGB4/JUP ratio (high-ITGB4/JUP) was revealed to be a primary contributor to distant metastasis without the involvement of clinicopathological factors, suggesting intervention of a critical step dependent on the function of the integrin $\beta 4$ subunit. Kaplan-Meier curves revealed positive margin as a lethal treatment consequence in high-ITGA3/CD9 and YK4 double-positive cases.

Conclusion: Two types of metastatic trait were found in OSCC: locoregional dissemination, which was reflected by high-ITGA3/CD9, and distant metastasis through hematogenous dissemination, uniquely distinguished by high-ITGB4/JUP. The clinical significance of the integrin biomarkers implies that biological mechanisms such as cancer cell motility and anchorage-independent survival are vital for OSCC recurrence and metastasis.
\end{abstract}

Keywords: Squamous cell carcinoma, Biomarker, Metastasis, Integrin alpha3, Integrin beta4

\section{Background}

Around 260000 new cases of oral cancer in the tongue, gingiva, oral floor, lip, and buccal mucosa are reported annually worldwide, and deaths from the disease reach approximately 127000 [1]. Squamous cell carcinoma of the oral cavity (OSCC) is the most prevalent malignancy of the head and neck region. Despite recent improvements

\footnotetext{
* Correspondence: nagata@dent.niigata-u.ac.jp

'Division of Oral and Maxillofacial Surgery, Niigata University Graduate School of Medical and Dental Sciences, Gakkocho-dori 2-5274, Chuo-ku, Niigata 951-8514, Japan

Full list of author information is available at the end of the article
}

in treatment, the survival of OSCC patients has not improved greatly over the past few decades [2]. Treatment failures of OSCC are primarily due to local and regional recurrence, and uncontrollable deaths can occur from distant metastasis [3,4], It is particularly important, therefore, to ensure a sufficient resection margin that takes the degree of infiltration into consideration [4-7]. While the rate of distant metastasis is less than $5 \%[7,8]$, there is no curable treatment once metastatic foci become visible. Another issue associated with OSCC treatment is a decline in the quality of life (QOL) of the patients because of unavoidable stomatognathic dysfunction [9]. To improve the

\section{Ciomed Central}


survival rate and patient QOL, it is essential to fully understand the risks of locoregional recurrence and distant metastasis. Histopathological features, immunohistological markers, blood biomarkers, and clinical features have been used as prognostic factors [10-12]; however, these parameters cannot provide relevant information during the early phases of treatment. Therefore, recent attempts to improve the diagnostic system have focused on gene mutations or polymorphisms and altered expression levels of biomarkers [13].

In our previous studies, we used microarray analysis and reverse transcription quantitative real time polymerase chain reaction (RT-QPCR) to report the potential use of integrin and tetraspanin family molecules as biomarkers for OSCC malignancy [14-16]. The integrin (ITG) molecule functions as a cell surface receptor that mediates extracellular mechanical and chemical signals into the cell interior, which modulates different signal transduction cascades. ITG also coordinates cell survival, apoptosis, proliferation, and motility and influences cell differentiation [17-20]. In the present study, we used RT-QPCR to determine the gene expression of integrin $\alpha 3$ (ITGA3) and integrin $\beta 4$ (ITGB4), as well as CD9 and plakoglobin (JUP), a desmosomal anchor protein gene [15]. We report here that certain ITGA3/CD9 and ITGB4/JUP gene expression ratios are specifically related to individual clinical events such as lymph node metastasis, primary site recurrence, distant metastasis, and uncontrollable death from OSCC.

\section{Methods}

\section{Patients and specimens}

Tumor samples for gene expression analyses were collected at the time of biopsy from 270 patients with OSCC who were treated at the Dental Department of Niigata University Medical and Dental Hospital, Niigata, Japan, the Special Dental Care and Oral Surgery of Shinshu University Hospital, Nagano, Japan, and the Division of Oral Surgery of Nagaoka Red Cross Hospital, Nagaoka, Japan from 1999 to 2008 (Table 1). The treatment modalities included local resection, composite resection (resection of a primary oral cancer, a portion of the oral floor and mandible, and reconstruction with tissue transplantation and neck dissection), and composite resection with radiation therapy with or without intravenous adjuvant chemotherapy.

This study was performed in accordance with the guidelines of the Declaration of Helsinki and the study protocol for this project was approved by the Research Ethics Committee of Niigata University Medical and Dental Hospital, the Ethics Committee of Shinshu University School of Medicine, and the Ethics Committee of Nagaoka Red Cross Hospital. A written letter of consent was processed after obtaining the patient informed consent to participate in this study.

\section{Total RNA extraction from carcinoma tissue}

Cancer tissue specimens were preserved by immersion in RNAlater solution (Ambion Inc., Austin, TX, USA) immediately after sampling. The extraction of total RNA was performed using the RNeasy Lipid Tissue Mini Kit (QIAGEN, Tokyo, Japan) after homogenization by TissueLyser LT (QIAGEN) in QIAzol Lysis Reagent according to the manufacturer's standard protocol. Synthesis of first-strand cDNA was performed by reverse transcription using total RNA $(0.2-1 \mu \mathrm{g})$ as a template (Super Script III, Life Technologies, Carlsbad, CA, USA).

Table 1 Clinicopathological data of $\mathbf{2 7 0}$ patients with oral squamous cell carcinoma

\begin{tabular}{|c|c|c|}
\hline \multicolumn{2}{|l|}{ Clinicopathological factor } & \multirow{2}{*}{$\begin{array}{c}\begin{array}{c}\text { No. of } \\
\text { patients (\%) }\end{array} \\
270\end{array}$} \\
\hline & Total & \\
\hline Observation period (days) & 61-2182 (average, 1253.79) & \\
\hline Age (years) & 21 - 92 (average, 66.70) & \\
\hline \multirow[t]{2}{*}{ Sex } & Male & $166(61.48)$ \\
\hline & Female & $104(38.52)$ \\
\hline \multirow[t]{5}{*}{ Tumor size $(\mathrm{mm})^{1}$} & 5-60 (average, 26.63) & \\
\hline & $\leq 20$ & $86(31.85)$ \\
\hline & $21-30$ & $97(35.93)$ \\
\hline & $31-40$ & $62(22.96)$ \\
\hline & $>40$ & $25(9.26)$ \\
\hline \multirow[t]{4}{*}{ Tumor status $^{2}$} & $\mathrm{~T} 1$ & $83(30.74)$ \\
\hline & $\mathrm{T} 2$ & $123(45.56)$ \\
\hline & $\mathrm{T} 3$ & $5(1.85)$ \\
\hline & $\mathrm{T} 4$ & $59(21.85)$ \\
\hline \multirow[t]{4}{*}{ Lymph node metastasis ${ }^{3}$} & pNO & 149 (55.19) \\
\hline & pN1 & $41(15.18)$ \\
\hline & pN2 & $80(29.63)$ \\
\hline & pN3 & $0(0)$ \\
\hline \multirow[t]{2}{*}{ Histologic grade $(Y K 4)^{4}$} & $1-3$ & $115(42.59)$ \\
\hline & $4 c-d$ & $155(57.41)$ \\
\hline \multirow[t]{2}{*}{ Surgical margin ${ }^{5}$} & Negative & $244(90.37)$ \\
\hline & Positive & $26(9.63)$ \\
\hline \multirow[t]{2}{*}{ Primary site recurrence } & Negative & $240(88.89)$ \\
\hline & Positive & $30(11.11)$ \\
\hline \multirow[t]{2}{*}{ Distant metastasis } & Negative & $256(94.81)$ \\
\hline & Positive & $14(5.19)$ \\
\hline \multirow[t]{2}{*}{ Death outcome ${ }^{6}$} & Alive & $233(86.30)$ \\
\hline & Dead & $37(13.70)$ \\
\hline
\end{tabular}

${ }^{1}$ Major width of the tumor. ${ }^{2}$ Tumor (T) category according to the International Union Against Cancer (UICC) TNM classification of malignant tumors of the lip and oral cavity. ${ }^{3}$ Lymph node $(\mathrm{pN})$ category determined by pathologic examination of a surgical specimen, according to the UICC TNM classification of malignant tumors of the lip and oral cavity. ${ }^{4}$ Histopathologic classification of oral squamous cell carcinoma (YK grade) according to Yamamoto et al., 1983 (10). ${ }^{5}$ Histological tumor status of the surgical margin. ${ }^{6}$ Death outcome from uncontrollable oral squamous cell carcinoma. 


\section{Gene expression analysis by quantitative real-time polymerase chain reaction}

RT-QPCR was performed on a Smart Cycler (Cepheid, Sunnyvale, CA, USA) using cDNA synthesized from the cancer specimens and TaqMan probes (TaqMan Gene expression Assays, Life Technologies) according to the following protocol: $600 \mathrm{~s}$ at $95^{\circ} \mathrm{C}$, followed by thermal cycles of $15 \mathrm{~s}$ at $95^{\circ} \mathrm{C}$ and $60 \mathrm{~s}$ at $60^{\circ} \mathrm{C}$ for the extension. Relative standard curves representing several 10-fold cDNA dilutions (1:10:100:1 000:10 000:100 000) from an OSCC tissue sample were used for the linear regression analysis of other samples. The manufacturer's TaqMan probe assay IDs are as follows: ITGA3: Hs00233722_m1; ITGB4: Hs01103172_g1; CD9 [NM_001769]: Hs01124027_m1; and JUP: Hs00158408_m1.

\section{Histopathological classification of OSCC}

Hematoxylin and eosin staining was conducted using 10\% formalin-fixed, paraffin-embedded sections of OSCC. Histopathological malignancy was examined based on the mode of invasion, as defined by a previous study [10] in which histopathological invasiveness was classified as Grade 1-4. We categorized YK4- as Grade 1-3 and YK4+ as Grade 4 (Figure 1).

\section{Statistical analysis}

Two integrin gene expression ratios, ITGA3/CD9 and ITGB4/JUP, were calculated for each of the 270 patients with OSCC (Table 1). Clinicopathological parameters were age, sex, tumor size, T category (UICC TNM Classification of Malignant Tumors), histopathological mode of invasion (YK4) [10], and positive margin (histological tumor positive at the surgical margin). Clinical events were lymph node metastasis (LNM) determined by histopathological examination of the surgical specimen, primary site recurrence after surgery (PSR), distant metastasis after surgical excision of the primary cancer (DM), and death from uncontrollable OSCC (DO). DM following locoregional failure was included as locoregional recurrence.

The influence of the two integrin gene expression ratios and clinicopathological parameters on LNM, PSR, DM, and DO were reviewed by univariate analysis (logrank test) to optimize the combination of variables for the following multivariate analysis (Table 2). Analyses by Cox proportional hazards model and KaplanMeier curve were performed for LNM, PSR, and DO (or disease-specific survival) as endpoint events (SPSS 18.0, IBM Japan, Tokyo, Japan). Durations to the events were calculated from the date of first visit to the date of neck dissection, diagnosis of recurrence, and date of OSCC death or final observation. $P$-values $\leq 0.05$ were assigned as the level of significance.

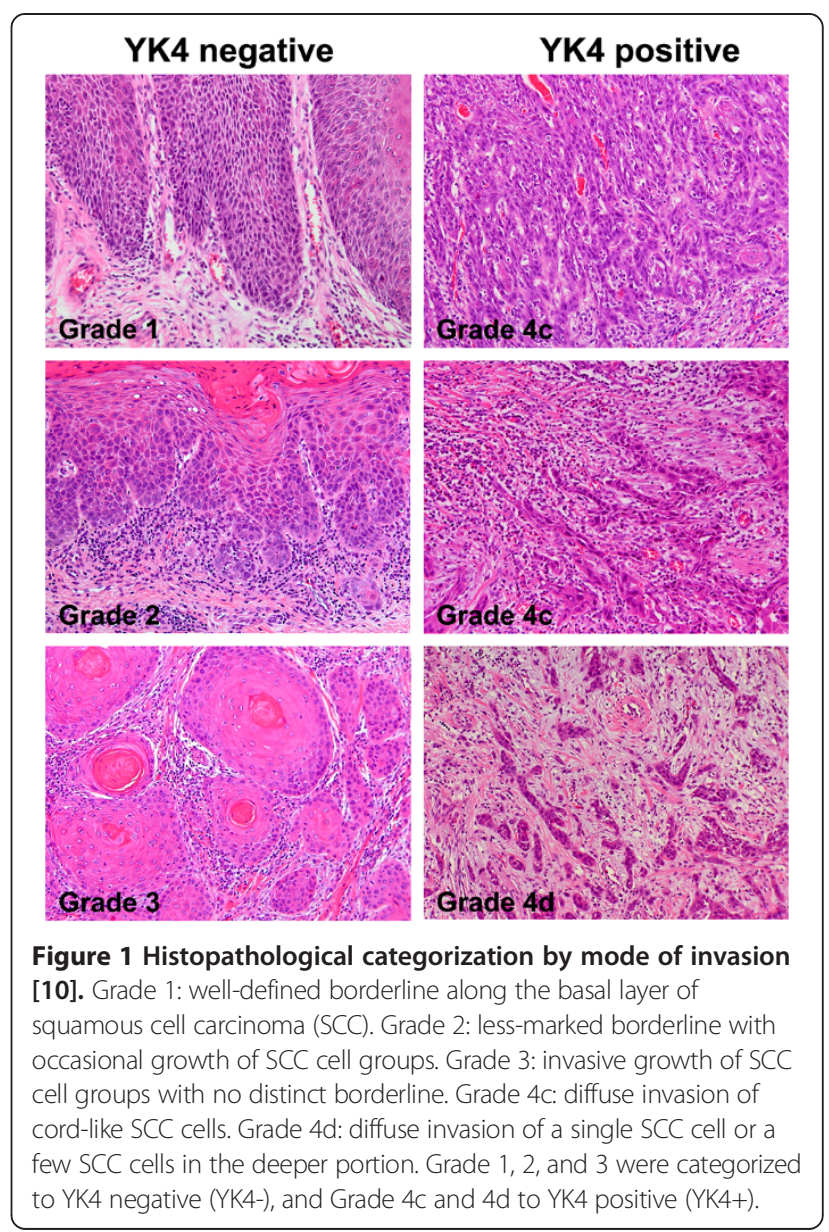

\section{Results}

Univariate analysis

The results of univariate analysis by the logrank test revealed the parameters that influenced the three clinical events and death outcome (DO) in each column of Table 2. Lymph node metastasis (LNM) was significantly associated with a high ITGA3/CD9 ratio (high-ITGA3/ CD9), tumor size and T3-4, which relate to the extent of tumor invasion, and histopathological mode of invasion (YK4). Primary site recurrence (PSR) was significantly associated with high-ITGA3/CD9, tumor size, T3-4 and positive margin, while distant metastasis was associated with high-ITGA3/CD9, a high ITGB4/JUP ratio (highITGB4/JUP), and YK4. DO was also associated with high-ITGA3/CD9 and high-ITGB4/JUP, and clinicopathological parameters with YK4 and positive margin.

\section{Lymph node metastasis}

In the Cox proportional hazards model of LNM, highITGA3/CD9, YK4, and the major width of the tumor (size) were reported as independently significant variables (Table 3A). The rate of LNM was represented by the Kaplan-Meier (K-M) curve (one minus cumulative 
Table 2 Logrank test (Mantel-Cox)

\begin{tabular}{|c|c|c|c|c|}
\hline & Lymph node metastasis & Primary site recurrence & Distant metastasis & OSCC death $^{7}$ \\
\hline \multicolumn{5}{|l|}{ Gene expression ratio } \\
\hline High-ITGA3/CD9 & 0.000 & 0.005 & 0.001 & 0.000 \\
\hline High-ITGB4/JUP & 0.022 & 0.237 & 0.000 & 0.001 \\
\hline \multicolumn{5}{|c|}{ Clinicopathological parameter } \\
\hline Age $^{1}$ & 0.449 & 0.321 & 0.207 & 0.365 \\
\hline Sex & 0.417 & 0.858 & 0.149 & 0.584 \\
\hline Size $^{2}$ & 0.000 & 0.007 & 0.703 & 0.084 \\
\hline $\mathrm{T} 3-4^{3}$ & 0.000 & 0.000 & 0.390 & 0.092 \\
\hline$Y K 4^{4}$ & 0.000 & 0.018 & 0.001 & 0.000 \\
\hline Positive margin ${ }^{5}$ & - - - & 0.000 & 0.427 & 0.000 \\
\hline \multicolumn{5}{|l|}{ Clinical event } \\
\hline Lymph node metastasis ${ }^{6}$ & -—- & 0.001 & 0.000 & 0.000 \\
\hline Primary site recurrence & -—- & -—— & 0.840 & 0.000 \\
\hline Distant metastasis & -——- & -——- & -——- & 0.000 \\
\hline
\end{tabular}

Numbers in the table show P-values for the parameters. ${ }^{1}$ Age was categorized into two groups $\geq 69$ and $<69$ based on the median age. ${ }^{2}$ Major width of the tumor was categorized into $>30 \mathrm{~mm}$ and $\leq 30 \mathrm{~mm} .{ }^{3} \mathrm{~T} 3$ to T4 of tumor (T) category according to the International Union Against Cancer (UICC) TNM classification of malignant tumors of the lip and oral cavity. ${ }^{4} 4 \mathrm{c}$ or $4 \mathrm{~d}$ of the histopathologic classification of oral squamous cell carcinoma according to Yamamoto et al., 1983 (10). ${ }^{5}$ Histological tumor positive of the surgical margin. ${ }^{6}$ Determined by pathologic examination of a surgical specimen. ${ }^{7}$ Death outcome from oral squamous cell carcinoma. Continuous variables of gene expression ratios are categorized according to the cut off points introduced by receiver operating characteristic curves. Median of ITGA3/CD9 and higher 30\% group of ITGB4/JUP are used for categorization.

survival) between groups of [high-ITGA3/CD9 and YK4]positive cases and the remaining (negative) cases according to size category strata (Figure 2a). The [high-ITGA3/CD9 and YK4]-positive cases consistently exhibited a higher rate of LNM (around 80\%) irrespective of the size strata, but the negative cases revealed an increasing rate of LNM with larger tumor size $(>30 \mathrm{~mm})$.

\section{Primary site recurrence}

Regarding primary site recurrence (PSR), the Cox proportional hazards model found high-ITGA3/CD9, T3-4 (TNM category) and positive margin to be independently significant variables (Table 3B). Although the positive margin is a sequential clinical event following surgery, it was involved in this analysis because of its considerable influence on PSR. The rate of PSR was represented by the K-M curves (one minus cumulative survival) between groups of [high-ITGA3/CD9 and T3-4]-positive cases and the remaining (negative) cases according to marginal status strata (Figure 2b). Risk of PSR was clearly enhanced in the positive group, indicating that positive margin is a remarkable causal event of PSR especially in [high-ITGA3/CD9 and T3-4]-positive cases.

\section{Distant metastasis}

In the Cox proportional hazards model on distant metastasis (DM), high-ITGB4/JUP and ITGA3/CD9 levels were reported as independently significant variables (Table 3C). In contrast to other clinical events, parameters related to tumor expansion, histopathological parameters, and LNM did not exhibit a significant influence in multivariate analysis, while high-ITGB4/JUP exhibited the strongest influence. The rate of DM was presented by the K-M curves (one minus cumulative survival) between the groups of [high-ITGA3/CD9 and high-ITGB4/JUP]-positive cases and remaining negative cases according to size category strata (Figure 2c). Among the 183 cases comprising "size $\leq 30 \mathrm{~mm}$ ", all of the nine cases that developed DM were extracted in 39 cases by a [high-ITGA3/CD9 and high-ITGB4/JUP]-double positive status. Among all 270 cases, 12 out of a total of 14 that developed DM were detected by a high-ITGA3/CD9 and high-ITGB4/JUP double-positive status. Both false positive cases had a tumor size of over $35 \mathrm{~mm}$, suggesting a higher diagnostic reliability for [high-ITGA3/CD9 and high-ITGB4/JUP] status in early OSCC.

\section{Death outcome from uncontrollable OSCC}

Regarding the OSCC death outcome (DO) Cox proportional hazards model, high-ITGA3/CD9, YK4, and positive margin were reported as independently significant variables (Table 3D). The cumulative survival was represented by the K-M curves between the groups of [highITGA3/CD9 and YK4]-positive cases and the remaining negative cases according to marginal status strata (Figure 2d). The risk of OSCC death was significantly higher in [high-ITGA3/CD9 and YK4]-positive cases, in which positive margin was a lethal treatment consequence in clinical outcome. 
Table 3 Cox proportional hazards model

\begin{tabular}{|c|c|c|c|c|c|c|c|}
\hline \multirow{3}{*}{$\begin{array}{l}\text { A } \\
\text { Variable }\end{array}$} & \multicolumn{7}{|c|}{ Lymph node metastasis } \\
\hline & \multirow{2}{*}{ B } & \multirow{2}{*}{ SE } & \multirow{2}{*}{ Wald } & \multirow{2}{*}{$P$} & \multirow{2}{*}{ OR } & \multicolumn{2}{|c|}{$95 \% \mathrm{Cl}$ for $\mathrm{OR}$} \\
\hline & & & & & & Lower limit & Upper limit \\
\hline High-ITGA3/CD9 & 1.063 & 0.203 & 27.545 & 0.000 & 2.896 & 1.947 & 4.307 \\
\hline YK4 ${ }^{1}$ & 0.961 & 0.216 & 19.887 & 0.000 & 2.614 & 1.714 & 3.989 \\
\hline $\operatorname{Size}^{2}$ & 0.622 & 0.186 & 11.126 & 0.001 & 1.862 & 1.292 & 2.684 \\
\hline B & \multicolumn{7}{|c|}{ Primary site recurrence } \\
\hline \multirow{2}{*}{ Variable } & \multirow{2}{*}{ B } & \multirow{2}{*}{ SE } & \multirow{2}{*}{ Wald } & \multirow{2}{*}{$P$} & \multirow{2}{*}{ OR } & \multicolumn{2}{|c|}{$95 \% \mathrm{Cl}$ for OR } \\
\hline & & & & & & Lower limit & Upper limit \\
\hline High-ITGA3/CD9 & 1.090 & 0.415 & 6.895 & 0.009 & 2.973 & 1.318 & 6.706 \\
\hline$T 3-4^{3}$ & 0.955 & 0.387 & 6.088 & 0.014 & 2.597 & 1.217 & 5.544 \\
\hline Positive margin $^{4}$ & 1.715 & 0.402 & 18.183 & 0.000 & 5.556 & 2.526 & 12.221 \\
\hline C & \multicolumn{7}{|c|}{ Distant metastasis } \\
\hline \multirow{2}{*}{ Variable } & \multirow{2}{*}{ B } & \multirow{2}{*}{ SE } & \multirow{2}{*}{ Wald } & \multirow{2}{*}{$P$} & \multirow{2}{*}{ OR } & \multicolumn{2}{|c|}{$95 \% \mathrm{Cl}$ for OR } \\
\hline & & & & & & Lower limit & Upper limit \\
\hline High-ITGB4/JUP & 2.311 & 0.771 & 8.990 & 0.003 & 10.088 & 2.227 & 45.704 \\
\hline High-ITGA3/CD9 & 2.108 & 1.047 & 4.051 & 0.044 & 8.233 & 1.057 & 64.135 \\
\hline $\mathrm{D}$ & \multicolumn{7}{|c|}{ Death outcome $\mathrm{s}^{5}$} \\
\hline \multirow{2}{*}{ Variable } & \multirow{2}{*}{$\mathrm{B}$} & \multirow{2}{*}{ SE } & \multirow{2}{*}{ Wald } & \multirow{2}{*}{ P } & \multirow{2}{*}{ OR } & \multicolumn{2}{|c|}{$95 \% \mathrm{Cl}$ for OR } \\
\hline & & & & & & Lower limit & Upper limit \\
\hline High-ITGA3/CD9 & 1.618 & 0.450 & 12.947 & 0.000 & 5.041 & 2.089 & 12.166 \\
\hline YK4 & 2.355 & 0.613 & 14.743 & 0.000 & 10.540 & 3.168 & 35.074 \\
\hline Positive margin & 1.906 & 0.398 & 22.954 & 0.000 & 6.725 & 3.084 & 14.665 \\
\hline
\end{tabular}

Cox Proportional Hazards Model for the risks of lymph node metastasis, primary site recurrence, distant metastasis, and OSCC death. ${ }^{1} 4 \mathrm{c}$ or $4 \mathrm{~d}$ of the histopathologic classification of oral squamous cell carcinoma according to Yamamoto et al., 1983 (10). ${ }^{2}$ Major width of the tumor was categorized into $>30$ mm and $\leq 30 \mathrm{~mm} .{ }^{3} \mathrm{~T} 3$ to T4 of tumor (T) category according to the International Union Against Cancer (UICC) TNM classification of malignant tumors of the lip and oral cavity. ${ }^{4} \mathrm{Histological}$ tumor positive of the surgical margin. ${ }^{5}$ Death outcome from uncontrollable oral squamous cell carcinoma. Continuous variables of gene expression ratios are categorized according to the cut off points introduced by receiver operating characteristic curves. Median of ITGA3/CD9 and higher $30 \%$ group of ITGB4/JUP are used for categorization. B, regression coefficient; SE, standard error; OR, odds ratio; $95 \% \mathrm{Cl}, 95 \%$ confidence interval.

\section{Discussion}

There are two types of OSCC metastatic trait. The first is simple lymph node metastasis (LNM) that can be locoregionally controlled, and the second is characterized by uncontrollable locoregional dissemination as well as distant metastasis (DM) through the blood circulation, leading to death. Distinguishing these two types of metastasis is difficult by current diagnostic procedures. In our previous study, we analyzed the ratio of expression of the 11 ITG family genes to that of the 14 functionally related genes; in total, 154 gene expression ratios for 66 tongue SCC cases [15]. We also investigated the potential of 45 tetraspanin family gene expression ratios that were calculated based on 6 tetraspanin family genes with housekeeping functionality or functionally related genes for 73 gingival SCC cases [16]. The results of these prior studies revealed two ITG gene expression ratios-those of ITGA3/ CD9 and ITGB4/JUP-as candidate biomarkers for OSCC.

Gene expression analysis using the entire tumor tissue is expected to involve several biases depending on cell composition, due to choice of sampling site, and degradation of molecules. Biopsy samples inevitably contain cell populations comprising cancer cells, cancer stroma cells such as fibroblasts, and inflammatory cells, and in some cases normal epithelial cells. However, we did not want to limit our analysis to the cancer cell population because we believe that analysis of the whole biopsy sample is essential for collecting practical information on the overall aspects of cancer biology that may contribute to the clinical behavior of the disease. For these reasons, we have focused on devising diagnostic gene expression ratios that are not affected by the contamination of normal cells or by sampling biases. To address this issue of biases, we have adopted a functional referencing strategy that uses gene expression data obtained by calculating gene pairs with relevance to intercellular localization and/or molecular function. As the consequence, we have demonstrated the practical benefits of ITGA3/CD9 and ITGB4/JUP in this study.

ITGA3/CD9 levels represent biological traits associated with lymphatic dissemination and local invasiveness. K-M curves for LNM showed that a [high-ITGA3/ 
a Lymph node metastasis
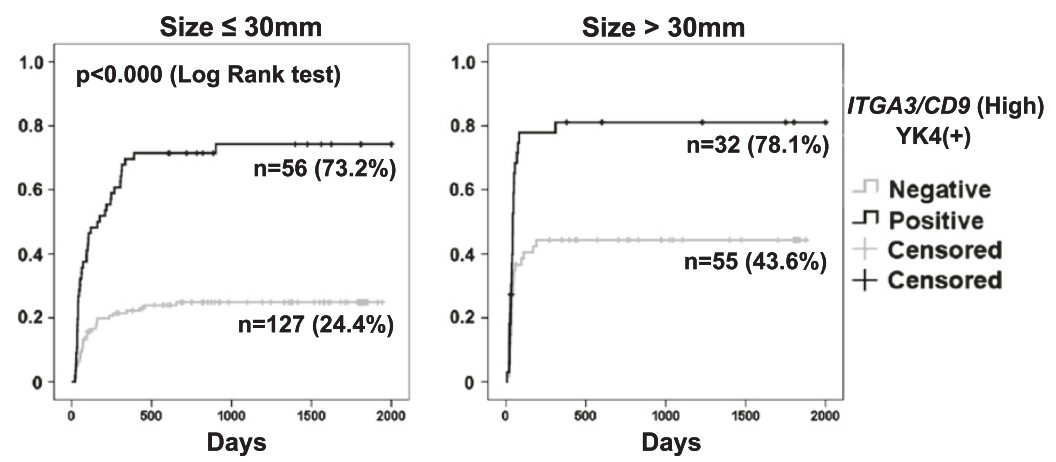

b

Primary site recurrence
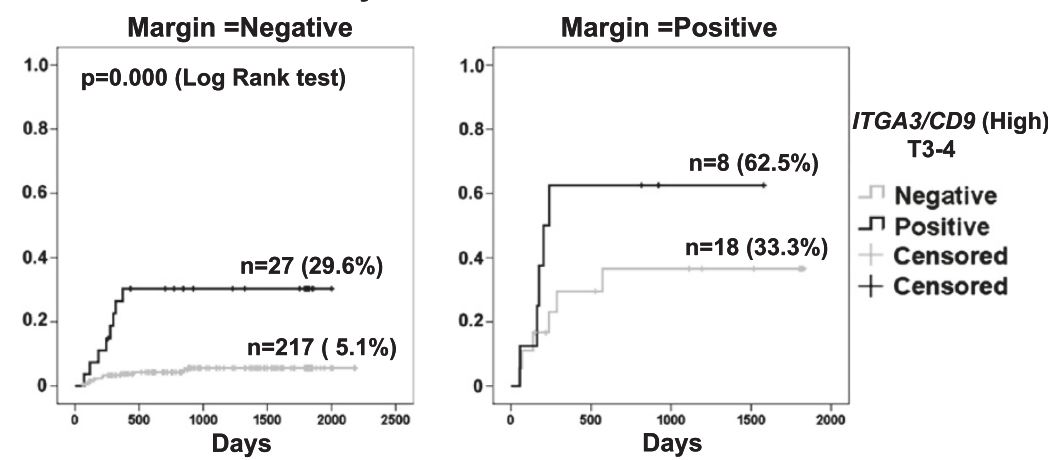

C
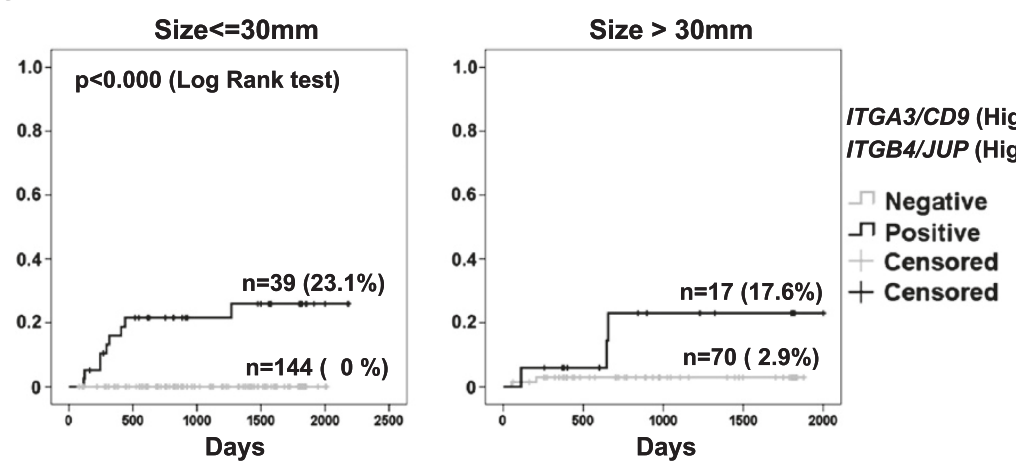

d

Disease-specific survival
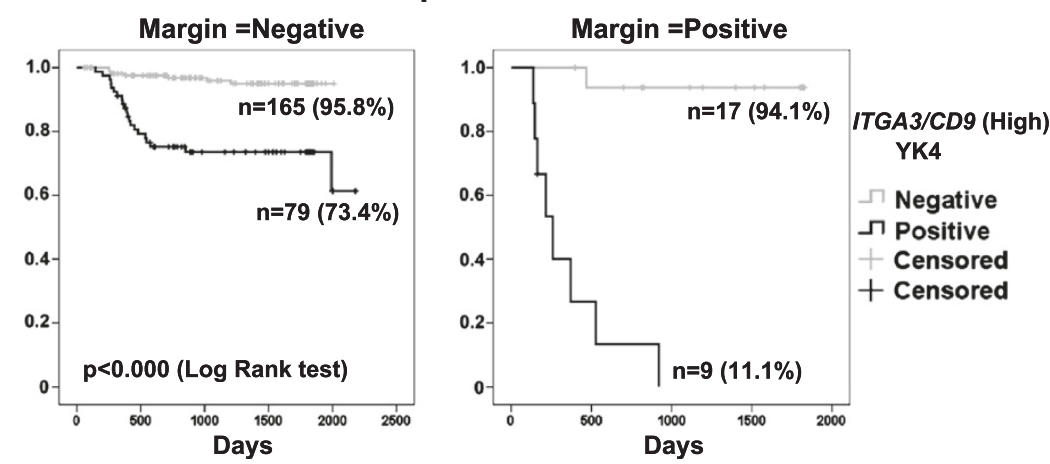

Figure 2 Kaplan-Meier survival curves for $\mathbf{2 7 0}$ patients with oral squamous cell carcinoma. Lymph node metastasis (a), primary site recurrence (b), distant metastasis (c), and disease-specific survival (d). Each consequence was stratified by a clinical event which effectively demonstrated influence of the factors. (a)-(c): Curves show 1 minus cumulative survival. (d): Curve shows cumulative survival. 


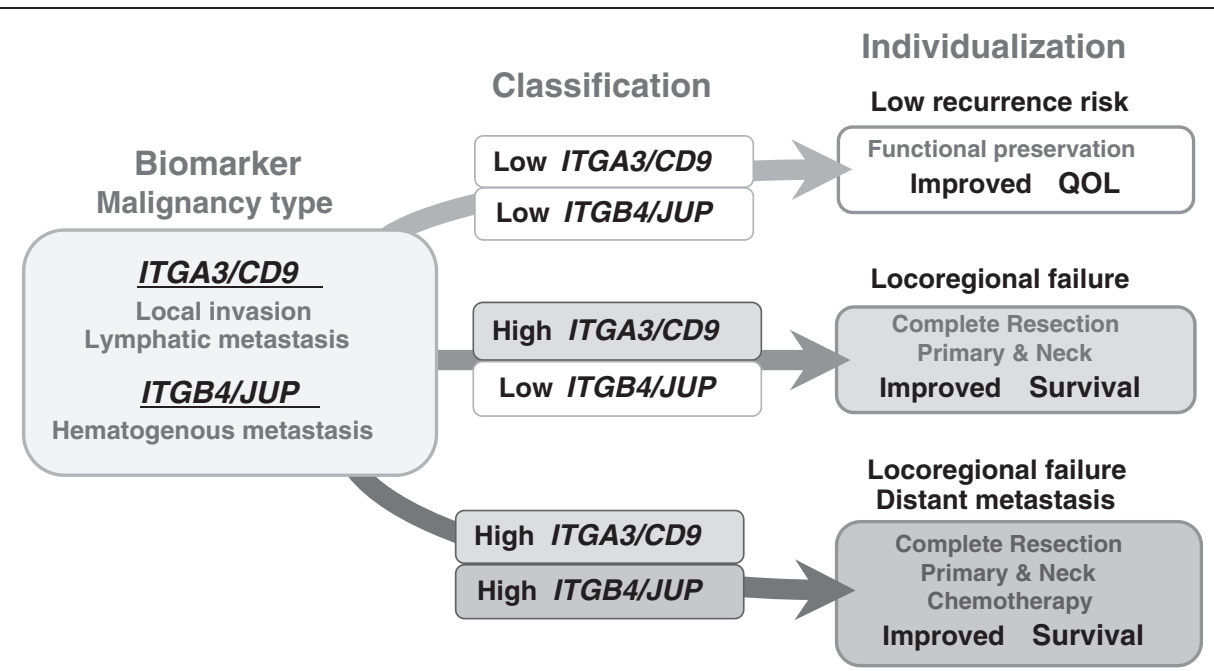

Figure 3 A hypothetical biomarker-oriented individualization of oral squamous cell carcinoma (OSCC) treatment based on the early diagnosis of OSCC malignancy type.

CD9 and YK4] status can identify highly metastatic cancer capable of early lymph node invasion (Figure 2a). High-ITGA3/CD9 and T3-4 were also reported to be related to primary site recurrence (PSR) (Table $3 \mathrm{~B}$ and Figure $2 \mathrm{~b}$ ), and positive margin is the most significant factor of PSR. However, only $42 \%$ of positive margin cases resulted in PSR, implying that biological traits are also critical in PSR. The $\alpha 3 \beta 1$ integrin complex is a major receptor for laminin 5 [17] and is involved in the maintenance of epithelial integrity, cell proliferation and motility, and survival of migrating keratinocytes through adhesion to extracellular matrix components [21-23]. CD9 is a tetraspanin family molecule, which forms tetraspanin webs (tetraspanin-enriched microdomains) by associating with various partner molecules such as integrins, growth factor receptors, and other tetraspanin molecules, to affect cell adhesion, signal transduction, proliferation, motility, differentiation, and cancer metastasis [16,24-28]. It has been reported that CD9 negatively influences cancer cell motility by regulating the re-organization of the actin cytoskeleton [29]. Collectively, it could be hypothesized that the ITGA3/CD9 gene expression ratio reflects the phase of cell motility and invasion in OSCC tissue.

In contrast to LNM and PSR, the ITGB4/JUP status exhibited a peculiar contribution for the prediction of distant metastasis (DM) (Table 3C), implying the involvement of a distinctive biological mechanism in DM. It is also characteristic of DM that no clinicopathological parameters were reported as contributing factors. These findings suggest the intervention of a critical step that depends on the function of the integrin $\beta 4$ subunit in the process of DM. $\alpha 6 \beta 4$ integrin is a transmembrane component of hemidesmosomes and functions mainly as a receptor for laminin 5 [30]. Plakoglobin $(J U P)$ is a component of the attachment plaque lining the cytoplasmic side of the desmosome to anchor intermediate filaments $[31,32]$. Since both the integrin $\beta 4$ subunit and JUP colocalize around the cell membrane, and mediate functions through cell adhesion, their expression ratio may reflect the oncological phase of SCC cells.

$\mathrm{DM}$ is rather rare in cancer-bearing conditions, despite the continuous release of numerous cancer cells into the circulation. This may be because most circulating cancer cells die without proliferating even after being implanted into distant tissues. In normal conditions, epithelial cells detached from the matrix or those that are attached via the wrong molecules undergo anchorage-related apoptosis. Therefore, acquirement of the ability for anchorageindependent survival, migration, and growth is essential for isolated tumor cells to engage in the process of hematogenous metastasis. Expression of integrin $\beta 4$ has been associated with tumor progression, aggressive behavior and poor prognosis in human malignant neoplasms [15,33-36]. It is reported that $\alpha 6 \beta 4$ integrin contributes to anchorage-independent growth through the ERK1/2 signaling pathway and to invasion through the combined activation of PI3K and Src [37]. Aberrant cytoplasmic localization of integrin $\beta 4$ in highly invasive OSCC cells suggests acquired anchorage-independent growth and motility through impaired ITGB4 expression [15].

\section{Conclusions}

The biomarker system of the ITGA3/CD9 and ITGB4/ JUP expression ratios may enable us precisely estimate the extent of local invasion and lymphatic metastasis, or hematogenous dissemination of OSCC. Information on 
ITGA3/CD9 level should enable surgeons to use an appropriate resection procedure to minimize the incidence of local recurrence as well as improve patient QOL by reducing oral dysfunction after treatment. The ITGB4/JUP level also provides information on the risk of distant metastasis, enabling effective pre- or postoperative adjuvant therapies to be given before metastatic lesions manifest (Figure 3). Recent genome-wide sequence studies have provided evidence that head and neck SCCs (HNSCCs), although morphologically similar, constitute distinct diseases at the molecular level. Since the major driver mutations accompanied by a large variety of genetic alternations are implicated in the carcinogenesis of SCC, it is thought to be impossible to assess malignancy type using only a few genetic markers. No genetic disruption in ITGA3, ITGB4, CD9, or JUP genes has been identified in reported HNSCC cases [38]. Therefore, changes in the ITGA3/CD9 and ITGB4/JUP levels as phenotypes due to a variety of mutations may serve as common indicators of biological malignancy of SCC. Further prospective clinical study will be indispensable for verifying the validity and clinical reliability of using gene expression ratios as a diagnostic means for distinguishing potential lymphatic and hematogenous disseminations. Likewise, biological involvement of the ITG molecules in locoregional invasion and hematogenous dissemination of OSCC remain to be determined.

\section{Abbreviations}

OSCC: Oral squamous cell carcinoma; QOL: Quality of life; RT-QPCR: Reverse transcription-quantitative real time polymerase chain reaction; LNM: Lymph node metastasis; PSR: Primary site recurrence; DM: Distant metastasis; DO: Death outcome; K-M curve: Kaplan-Meier curve.

\section{Competing interests}

There are no competing interests to declare.

\section{Authors' contributions}

MN, AAN, TKo, and KU carried out gene expression analysis and immunohistochemistry. MN, KU, TK, HH, KT, HK, NI, TO, and $\mathrm{MO}$ took charge of acquisition of the tumor sample and clinical data. MN and NK participated in the design of the study and performed statistical analysis. MN, KS, HK, and SS summarized and interpreted the data. MN wrote the paper; KS, RT, and TKa were involved in critically revising the manuscript for important intellectual content. All authors have read and approved the final manuscript.

\section{Acknowledgements}

This work was supported by the Japan Society for the Promotion of Science (Project No. 20592354), and by the Japan Science and Technology Agency, Adaptable \& Seamless Technology Transfer Program, Feasibility Study Stage (Project No. AS242Z03113P).

\footnotetext{
Author details

'Division of Oral and Maxillofacial Surgery, Niigata University Graduate School of Medical and Dental Sciences, Gakkocho-dori 2-5274, Chuo-ku, Niigata 951-8514, Japan. ${ }^{2}$ Department of Gastroenterology, Niigata University Graduate School of Medical and Dental Sciences, Asahimachi-dori 1-757, Chuo-ku, Niigata 951-8510, Japan. ${ }^{3}$ Department of Dentistry and Oral Surgery, Shinshu University School of Medicine, Asahi 3-1-1, Matsumoto 390-8621, Japan. ${ }^{4}$ Division of Dental Clinic and Oral Surgery, Nagaoka Red Cross Hospital, Terashimamachi 297-1, Nagaoka 940-2085, Japan. ${ }^{5}$ Department of Medical Informatics, Niigata University Medical \& Dental Hospital, Asahimachi-dori 1-754, Chuo-ku, Niigata 951-8520, Japan. ${ }^{6}$ Department of Oral and Maxillofacial Surgery, Kyoto University Graduate
}

School of Medicine, Shogoin-Kawahara-cho, Sakyo-ku, Kyoto 606-8507, Japan. ${ }^{7}$ Division of Oral Bioengineering, Department of Tissue Regeneration and Reconstitution, Niigata University Graduate School of Medical and Dental Sciences, Gakkocho-dori 2-5274, Chuo-kuNiigata 951-8514, Japan. ${ }^{8}$ Division of Reconstructive Surgery for Oral and Maxillofacial Region, Niigata University Graduate School of Medical and Dental Sciences, Gakkocho-dori 2-5274,

Chuo-ku, Niigata 951-8514, Japan.

Received: 5 February 2013 Accepted: 30 August 2013

Published: 5 September 2013

\section{References}

1. Ferlay J, Shin HR, Bray F, Forman D, Mathers C, Parkin DM: Estimates of worldwide burden of cancer in 2008: GLOBOCAN 2008. Int J Cancer 2010, 127:2893-2917.

2. Yako-Suketomo H, Matsuda T: Comparison of time trends in lip, oral cavity and pharynx cancer mortality (1990-2006) between countries based on the WHO mortality database. Jpn J Clin Oncol 2010, 40:1118-1119.

3. Jerjes W, Upile T, Petrie A, Riskalla A, Hamdoon Z, Vourvachis M, Karavidas K, Jay A, Sandison A, Thomas GJ, Kalavrezos N, Hopper C: Clinicopathological parameters, recurrence, locoregional and distant metastasis in 115 T1-T2 oral squamous cell carcinoma patients. Head Neck Oncol 2010, 2:9.

4. Sessions DG, Lenox J, Spector GJ, Chao C, Chaudry OA: Analysis of treatment results for base of tongue cancer. Laryngoscope 2003, 113:1252-1261.

5. Slootweg PJ, Hordijk GJ, Schade Y, van Es RJ, Koole R: Treatment failure and margin status in head and neck cancer. A critical view on the potential value of molecular pathology. Oral Oncol 2002, 38:500-503.

6. Weijers M, Snow GB, Bezemer DP, van der Wal JE, van der Waal I: The status of the deep surgical margins in tongue and floor of mouth squamous cell carcinoma and risk of local recurrence; an analysis of 68 patients. Int J Oral Maxillofac Surg 2004, 33:146-149.

7. Ganly I, Patel S, Shah J: Early stage squamous cell cancer of the oral tongueclinicopathologic features affecting outcome. Cancer 2012, 118:101-111.

8. León X, Quer M, Orús C, del Prado Venegas M, López M: Distant metastases in head and neck cancer patients who achieved locoregional control. Head Neck 2000, 22:680-686.

9. Infante-Cossio P, Torres-Carranza E, Cayuela A, Hens-Aumente E, Pastor-Gaitan P, Gutierrez-Perez JL: Impact of treatment on quality of life for oral and oropharyngeal carcinoma. Int J Oral Maxillofac Surg 2009, 38:1052-1058.

10. Yamamoto E, Kohama G, Sunakawa H, Iwai M, Hiratsuka H: Mode of invasion, bleomycin sensitivity, and clinical course in squamous cell carcinoma of the oral cavity. Cancer 1983, 51:2175-2180.

11. Rahima B, Shingaki S, Nagata M, Saito C: Prognostic significance of perineural invasion in oral and oropharyngeal carcinoma. Oral Surg Oral Med Oral Pathol Oral Radiol Endod 2004, 97:423-431.

12. Bello IO, Soini Y, Salo T: Prognostic evaluation of oral tongue cancer: means, markers and perspectives (I). Oral Oncol 2010, 46:630-635.

13. Bello IO, Soini Y, Salo T: Prognostic evaluation of oral tongue cancer: means, markers and perspectives (II). Oral Oncol 2010, 46:636-643.

14. Nagata M, Fujita H, Ida H, Hoshina H, Inoue T, Seki Y, Ohnishi M, Ohyama T, Shingaki S, Kaji M, Saku T, Takagi R: Identification of potential biomarkers of lymph node metastasis in oral squamous cell carcinoma by CDNA microarray analysis. Int J Cancer 2003, 106:683-689.

15. Kurokawa A, Nagata M, Kitamura N, Noman AA, Ohnishi M, Ohyama T, Kobayashi T, Shingaki S, Takagi R: Diagnostic value of integrin alpha3, beta4, and beta5 gene expression levels for the clinical outcome of tongue squamous cell carcinoma. Cancer 2008, 112:1272-1281.

16. Hirano C, Nagata M, Noman AA, Kitamura N, Ohnishi M, Ohyama T, Kobayashi T, Suzuki K, Yoshizawa M, Izumi N, Fujita H, Takagi R: Tetraspanin gene expression levels as potential biomarkers for malignancy of gingival squamous cell carcinoma. Int J Cancer 2009, 124:2911-2916.

17. Plow EF, Haas TA, Zhang L, Loftus J, Smith JW: Ligand binding to integrins. J Biol Chem 2000, 275:21785-21788

18. Calderwood DA, Shattil SJ, Ginsberg MH: Integrins and actin filaments: reciprocal regulation of cell adhesion and signaling. J Biol Chem 2000, 275:22607-22610.

19. Stupack DG, Cheresh DA: Get a ligand, get a life: integrins, signaling and cell survival. J Cell Sci 2002, 115:3729-3738.

20. Danen $E H$, Sonnenberg A: Integrins in regulation of tissue development and function. J Pathol 2003, 200:471-480. 
21. DiPersio CM, Hodivala-Dilke KM, Jaenisch R, Kreidberg JA, Hynes RO: alpha3beta1 Integrin is required for normal development of the epidermal basement membrane. J Cell Biol 1997, 137:729-742.

22. Gonzales M, Haan K, Baker SE, Fitchmun M, Todorov I, Weitzman S, Jones JC: A cell signal pathway involving laminin-5, alpha3beta1 integrin, and mitogen-activated protein kinase can regulate epithelial cell proliferation. Mol Biol Cell 1999, 10:259-270.

23. Manohar A, Shome SG, Lamar J, Stirling L, lyer V, Pumiglia K, DiPersio CM: Alpha 3 beta 1 integrin promotes keratinocyte cell survival through activation of a MEK/ERK signaling pathway. J Cell Sci 2004, 117:4043-4054.

24. Miyamoto S, Maruyama A, Okugawa K, Akazawa K, Baba H, Maehara Y, Mekada E: Loss of motility-related protein 1 (MRP1/CD9) and integrin alpha3 expression in endometrial cancers. Cancer 2001, 92:542-548.

25. Hashida H, Takabayashi A, Tokuhara T, Taki T, Kondo K, Kohno N, Yamaoka Y, Miyake M: Integrin alpha3 expression as a prognostic factor in colon cancer: association with MRP-1/CD9 and KAI1/CD82. Int J Cancer 2002, 97:518-525.

26. Levy S, Shoham T: Protein-protein interactions in the tetraspanin web. Physiology (Bethesda) 2005, 20:218-224.

27. Lazo PA: Functional implications of tetraspanin proteins in cancer biology. Cancer Sci 2007, 98:1666-1677.

28. Sridhar SC, Miranti CK: Tetraspanin KAI1/CD82 suppresses invasion by inhibiting integrin-dependent crosstalk with c-Met receptor and Src kinases. Oncogene 2006, 25:2367-2378.

29. Huang CL, Ueno M, Liu D, Masuya D, Nakano J, Yokomise H, Nakagawa T, Miyake M: MRP-1/CD9 gene transduction regulates the actin cytoskeleton through the downregulation of WAVE2. Oncogene 2006, 25:6480-6488.

30. Nievers MG, Schaapveld RQ, Sonnenberg A: Biology and function of hemidesmosomes. Matrix Biol 1999, 18:5-17.

31. Kowalczyk AP, Bornslaeger EA, Borgwardt JE, Palka HL, Dhaliwal AS, Corcoran CM, Denning MF, Green KJ: The amino-terminal domain of desmoplakin binds to plakoglobin and clusters desmosomal cadherinplakoglobin complexes. J Cell Biol 1997, 139:773-784.

32. Fausser JL, Schlepp O, Aberdam D, Meneguzzi G, Ruch JV, Lesot H: Localization of antigens associated with adherens junctions, desmosomes, and hemidesmosomes during murine molar morphogenesis. Differentiation 1998, 63:1-11.

33. Gleason B, Adley B, Rao MS, Diaz LK: Immunohistochemical detection of the beta4 integrin subunit in pancreatic adenocarcinoma. $J$ Histochem Cytochem 2005, 53:799-801.

34. Brendle A, Lei H, Brandt A, Johansson R, Enquist K, Henriksson R, Hemminki K, Lenner P, Försti A: Polymorphisms in predicted microRNA-binding sites in integrin genes and breast cancer: ITGB4 as prognostic marker. Carcinogenesis 2008, 29:1394-1399.

35. Lu S, Simin K, Khan A, Mercurio AM: Analysis of integrin beta4 expression in human breast cancer: association with basal-like tumors and prognostic significance. Clin Cancer Res 2008, 14:1050-1058.

36. Wan X, Kim SY, Guenther LM, Mendoza A, Briggs J, Yeung C, Currier D, Zhang H, Mackall C, Li WJ, Tuan RS, Deyrup AT, Khanna C, Helman L: Beta4 integrin promotes osteosarcoma metastasis and interacts with ezrin. Oncogene 2009, 28:3401-3411.

37. Dutta U, Shaw LM: A key tyrosine (Y1494) in the beta4 integrin regulates multiple signaling pathways important for tumor development and progression. Cancer Res 2008, 68:8779-8787.

38. Stransky N, Egloff AM, Tward AD, Kostic AD, Cibulskis K, Sivachenko A, Kryukov GV, Lawrence MS, Sougnez C, McKenna A, Shefler E, Ramos AH, Stojanov P, Carter SL, Voet D, Cortés ML, Auclair D, Berger MF, Saksena G, Guiducci C, Onofrio RC, Parkin M, Romkes M, Weissfeld JL, Seethala RR, Wang L, Rangel-Escareño C, Fernandez-Lopez JC, Hidalgo-Miranda A, Melendez-Zajgla J, et al: The mutational landscape of head and neck squamous cell carcinoma. Science 2011, 333:1157-1160.

doi:10.1186/1471-2407-13-410

Cite this article as: Nagata et al: ITGA3 and ITGB4 expression biomarkers estimate the risks of locoregional and hematogenous dissemination of oral squamous cell carcinoma. BMC Cancer 2013 13:410.

\section{Submit your next manuscript to BioMed Central and take full advantage of:}

- Convenient online submission

- Thorough peer review

- No space constraints or color figure charges

- Immediate publication on acceptance

- Inclusion in PubMed, CAS, Scopus and Google Scholar

- Research which is freely available for redistribution

Submit your manuscript at www.biomedcentral.com/submit
C Biomed Central 